\title{
X-ray emission from the stellar population in M 32
}

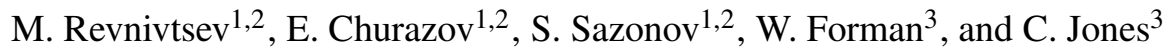 \\ 1 Max-Planck-Institute für Astrophysik, Karl-Schwarzschild-Str. 1, 85740 Garching bei München, Germany \\ e-mail: mikej@mpa-garching.mpg. de \\ 2 Space Research Institute, Russian Academy of Sciences, Profsoyuznaya 84/32, 117997 Moscow, Russia \\ 3 Harvard-Smithsonian Center for Astrophysics, 60 Garden Street, Cambridge, MA 02138, USA
}

Received 21 February 2007 / Accepted 22 July 2007

\section{ABSTRACT}

\begin{abstract}
Using Chandra observations, we study the X-ray emission of the stellar population in the compact dwarf elliptical galaxy M 32 . The proximity of M 32 allows one to resolve all bright point sources with luminosities higher than $8 \times 10^{33} \mathrm{erg} \mathrm{s}^{-1}$ in the $0.5-7 \mathrm{keV}^{-}$band. The remaining (unresolved) emission closely follows the galaxy's optical light and is characterized by an emissivity per unit stellar mass of $\sim 4.3 \times 10^{27} \mathrm{erg} \mathrm{s}^{-1} M_{\odot}^{-1}$ in the $2-10 \mathrm{keV}$ energy band. The spectrum of the unresolved emission above a few keV smoothly joins the X-ray spectrum of the Milky Way's ridge measured with RXTE and INTEGRAL. These results strongly suggest that weak discrete X-ray sources (accreting white dwarfs and active binary stars) provide the bulk of the "diffuse" emission of this gas-poor galaxy. Within the uncertainties, the average X-ray properties of the M 32 stars are consistent with those of the old stellar population in the Milky Way. The inferred cumulative soft X-ray $(0.5-2 \mathrm{keV})$ emissivity is however smaller than is measured in the immediate Solar vicinity in our Galaxy. This difference is probably linked to the contribution of young (age « $1 \mathrm{Gyr}$ ) stars, which are abundant in the Solar neighborhood but practically absent in M 32. Combining Chandra, RXTE and INTEGRAL data, we obtain a broad-band $(0.5-60 \mathrm{keV}) \mathrm{X}$-ray spectrum of the old stellar population in galaxies.
\end{abstract}

Key words. ISM: general - galaxies: dwarf - galaxies: individual: M 32 - galaxies: general - galaxies: stellar content X-rays: diffuse background

\section{Introduction}

Normal galaxies (i.e. those lacking a bright active nucleus) are often observed as X-ray bright objects. In spiral galaxies, the main contributors to the $\mathrm{X}$-ray emission are the populations of low- and high-mass X-ray binaries (LMXBs and HMXBs, see e.g. Fabbiano \& White 2003; Gilfanov 2004, for reviews). Elliptical galaxies lack HMXBs associated with recent star formation episodes, but instead contain large quantities of hot $(\sim 0.5 \mathrm{keV})$ interstellar gas (e.g. Forman et al. 1985; Canizares et al. 1987). The amount of hot gas correlates with the galaxy mass, although with considerable scatter, and in massive systems the hot gas provides the bulk of the X-ray emission.

In the Milky Way, apart from the emission from LMXBs and HMXBs an additional component is clearly seen, spatially concentrated to the Galactic plane - the so-called Galactic ridge X-ray emission (GRXE, e.g. Worrall et al. 1982). The luminosity of this component amounts to only a few per cent of the total X-ray luminosity of the Milky Way, dominated by bright LMXBs and HMXBs. The origin of this component remained controversial for two decades, until recently a convincing body of evidence showed that the bulk of the GRXE is produced collectively by millions of weak X-ray sources mostly belonging to the old stellar population of the Galaxy - cataclysmic variables (CVs) and coronally active stars in close binaries (active binaries, or ABs) (Revnivtsev et al. 2006; Sazonov et al. 2006; Krivonos et al. 2006; Revnivtsev et al. 2007). The X-ray luminosities of most of these sources are less than $10^{31} \mathrm{erg} \mathrm{s}^{-1}$, which makes the resolution of the GRXE into discrete sources a challenging (although not completely infeasible) problem.

Recently, the cumulative X-ray emissivity of faint Galactic point sources per unit stellar mass has been directly determined (by integrating the $\mathrm{X}$-ray luminosity function from $\sim 10^{27}$ to $\sim 10^{34} \mathrm{erg} \mathrm{s}^{-1}$ ) using an X-ray selected sample of sources located in the vicinity of the Sun (Sazonov et al. 2006). However, given the small size of this sample and possible pecularities of the solar neighborhood, the resulting emissivity estimate may differ from the average value for the Galactic stellar population by a significant factor of $\sim 2$. It is very important to further reduce this uncertainty in order to put a firm upper limit on any truly diffuse contribution to the GRXE. This is particularly important for understanding the physical processes taking place in the interstellar medium. Unfortunately, the presently available data for the Milky Way do not allow a considerable improvemement of the situation.

One could hope to directly measure the cumulative X-ray emissivity of stellar populations in other galaxies, provided they contain very little hot interstellar gas and the contribution of bright LMXBs and HMXBs can be completely resolved. Such a measurement, in particular, would provide us important information about the cumulative soft X-ray emission (below $\sim 2 \mathrm{keV}$ ) of stellar sources, which is practically inaccessible in our Galaxy due to the strong interstellar absorption through the Milky Way.

The best objects for performing this kind of measurement are dwarf elliptical galaxies of the Local Group. Indeed, they i) lack HMXBs and ii) they are close to us, which makes it possible to resolve and subtract the contribution of virtually all LMXBs using the Chandra telescope ${ }^{1}$. Also, their shallow potential wells contain very little (if any) hot, X-ray emitting gas.

\footnotetext{
${ }^{1}$ The critical property here is the ability of the telescope to resolve all sources with luminosities $L_{\mathrm{x}}>10^{35} \mathrm{erg} \mathrm{s}^{-1}$, since at lower luminosities the contribution of LMXBs becomes small. For a distance of $1 \mathrm{Mpc}$ Chandra needs $\sim 20$ ks to achieve this sensitivity.
} 

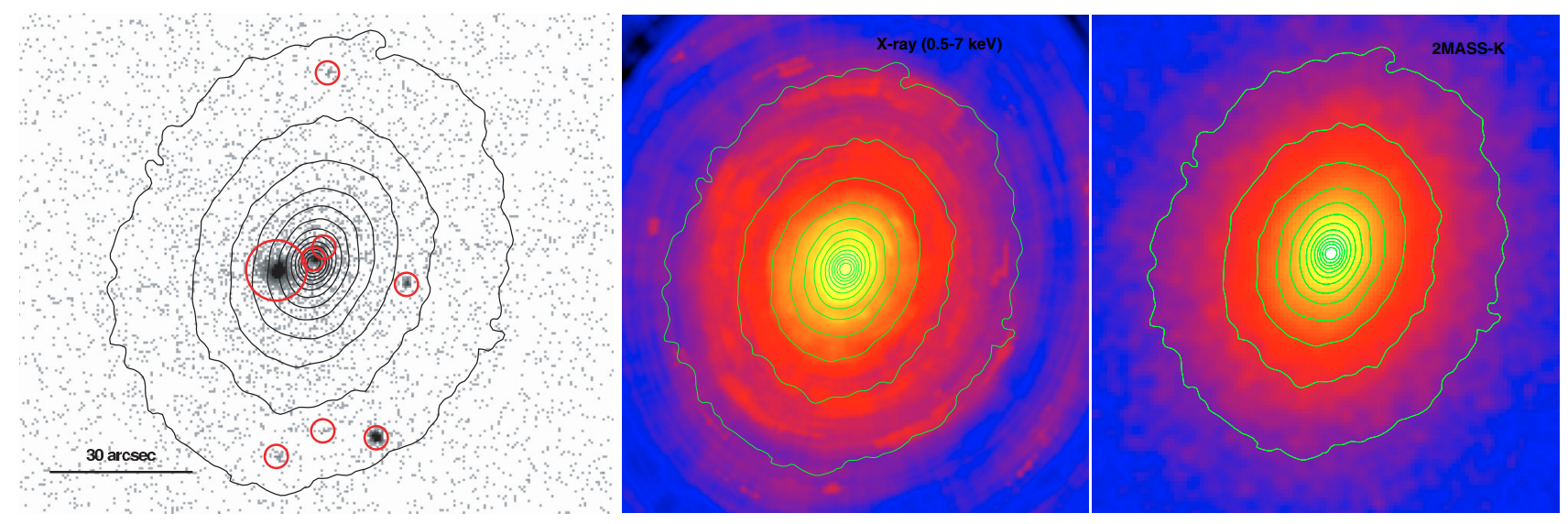

Fig. 1. Left - raw Chandra image of M 32 in the energy band $0.3-7 \mathrm{keV}$. Contours denote isophotes of the galaxy in near-infrared light. Circles show positions of detected sources. Center - adaptively smoothed Chandra image of M 32 in the 0.5-7 keV band with detected point sources removed. Contours denote isophotes of the galaxy in near-infrared light ( $K$-band, see right panel). Right $-K$-band image of the galaxy from the 2MASS survey.

With these considerations in mind, we selected for a pilot study the nearby - $805 \mathrm{kpc}$ (Mateo 1998) - dwarf elliptical galaxy M32. This is a well-known object, which has been extensively studied in practically all wave-bands (Mateo 1998). It has a total stellar mass of $\sim 10^{9} M_{\odot}$, contains very little cold gas (Welch \& Sage 2001), and presents no evidence for recent star formation activity (REFS). The total M 32 X-ray luminosity $\left(6-8 \times 10^{37} \mathrm{erg} \mathrm{s}^{-1}\right)$ is dominated by a single source, most likely a LMXB, M 32 X-3 (e.g. Loewenstein et al. 1998; Ho et al. 2003). Emission from the nucleus of the galaxy and a supersoft X-ray source have also been detected (Ho et al. 2003).

Previous analyses of Chandra observations of M32 revealed extended X-ray emission in addition to the detected point sources (Ho et al. 2003; Fukazawa et al. 2006). Ho et al. (2003) proposed that this extended emission is produced by hot interstellar gas in the galaxy.

We argue below that the unresolved X-ray emission in M 32 is probably a superposition of numerous faint point $X$-ray sources in the galaxy, mostly CVs and ABs, and has essentially the same nature and properties as the GRXE in the Milky Way.

The structure of the paper is as follows: in Sect. 2 we describe the data used in the analysis, in Sect. 3 we present the obtained results on the morphology and spectrum of the unresolved emission in M 32, in Sect. 4 we discuss possible origins of the unresolved emission, and argue that the most plausible explanation of this emission is the cumulative contribution of weak unresolved sources of the old stellar population in M 32, at the end of Sect. 4 we discuss some predictions for future observations of M 32 and other gas-poor elliptical galaxies, and we conclude in Sect. 5.

\section{Data analysis}

We used all publicly available Chandra observations of M 32 in which the nucleus of the galaxy was far from the gaps between the chips. Specifically, we analyzed observations with OBSIDs 313, 314, 1576, 1580, 1584, 2017, 2494, 2894, 2899, and 5690 . The total exposure time of our dataset is $\sim 215 \mathrm{ks}$.

The Chandra data were reduced following a standard procedure fully described in Vikhlinin et al. (2005). The background for the spectral analysis of the extended emission of M 32 was obtained from a $3^{\prime}$ by $1.5^{\prime}$ rectangle at $\sim 2^{\prime}$ to the south of the center of the galaxy.
Point sources were detected using the wavelet decomposition package wvdecomp of ZHTOOLS (Vikhlinin et al. $1998)^{2}$. The source detection threshold for the analyzed dataset is $\sim 10^{-16} \mathrm{erg} \mathrm{s}^{-1} \mathrm{~cm}^{-2}$ (in the energy band $0.5-7 \mathrm{keV}$ ), which corresponds to a source luminosity $\sim 8 \times 10^{33} \mathrm{erg} \mathrm{s}^{-1}(0.5-7 \mathrm{keV})$ at the M 32 distance.

\section{Results}

\subsection{Morphology}

Figure 1 shows the $2^{\prime}$ by $2^{\prime}$ raw Chandra image of M 32 in the $0.3-7 \mathrm{keV}$ energy band. Within a radius of $50^{\prime \prime}$ around the center of the galaxy we detected 8 point sources (including the galactic nucleus), 3 of which (within 12" of the nucleus) were previously reported by Ho et al. (2003) based on a shorter $50 \mathrm{ks}$ exposure. The detected sources were excluded from the analysis of the diffuse emission. In Fig. 1 (middle panel) an adaptively smoothed X-ray image of M 32 without point sources is shown with the near-infrared isophotes from the 2MASS $K$-band image superposed.

In the optical and near-infrared bands, M 32 has a nearly elliptical shape with ellipticity $r_{\text {major }} / r_{\text {minor }} \sim 1.17$ (see e.g. Kent 1987). In order to construct radial profiles of the surface brightness of the galaxy's extended (unresolved) X-ray emission, we extracted fluxes in elliptical annuli defined by the above value of ellipticity and the position angle derived from the isophotes of the galaxy in near-infrared light (Fig. 1).

The resulting radial surface-brightness profiles in two energy bands, $0.5-2 \mathrm{keV}$ and $2-7 \mathrm{keV}$, are shown in Fig. 2 together with the near-infrared ( $K$-band) light profile, derived from the 2MASS image of M32 using the same elliptical annuli. Clearly the X-ray intensity in both bands closely follows the near-infrared surface brightness. The X-ray profile can be well fit by a de Vaucouleurs law with effective radius $r_{\text {eff, }}$ major $=35 \pm 3^{\prime \prime}$. This value is fully compatible with that obtained from optical imaging (see e.g. Kent 1987, and also Fig. 2 which compares the X-ray and optical light profiles).

The constancy and value of the hardness ratio further support the association of the unresolved X-ray emission with the stellar light and its associated point sources. The hardness of the $\mathrm{X}$-ray spectrum (the ratio of the $2-7$ and $0.5-2 \mathrm{keV}$ fluxes) is

\footnotetext{
${ }^{2}$ http://hea-www.harvard.edu/saord/zhtools/
} 


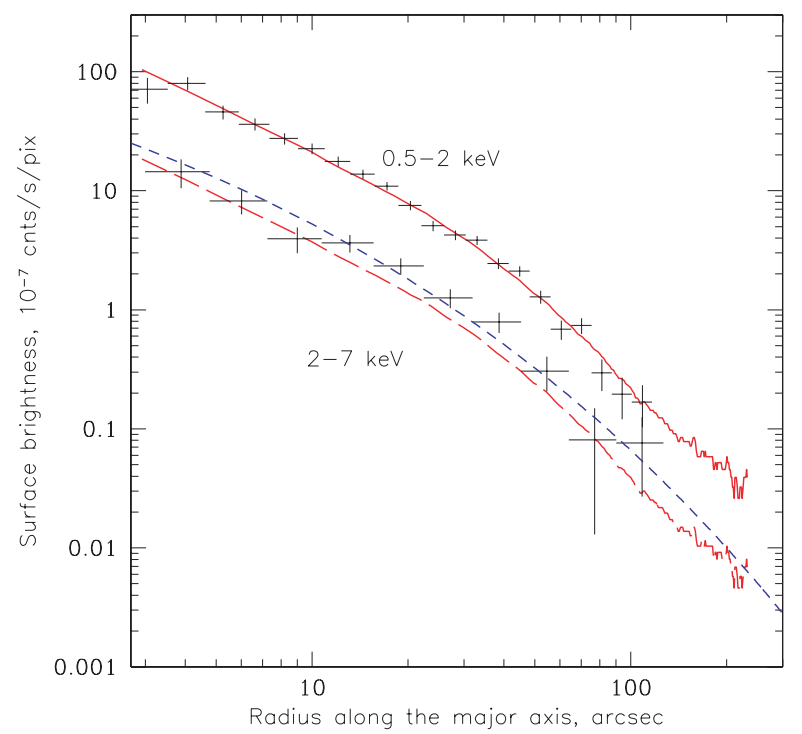

Fig. 2. Radial surface brightness profiles of M32 in two X-ray energy bands (crosses). The contribution of point sources with luminosities $>10^{34} \mathrm{erg} \mathrm{s}^{-1}$ has been removed. The solid and long-dashed lines show the shape of the M32 profile in the near-infrared $K$-band (arbitrary normalizations). The short-dashed line shows the de Vaucouleurs law with core radius $r_{\mathrm{c} \text {,major }}=35^{\prime \prime}$ (arbitrary normalization).

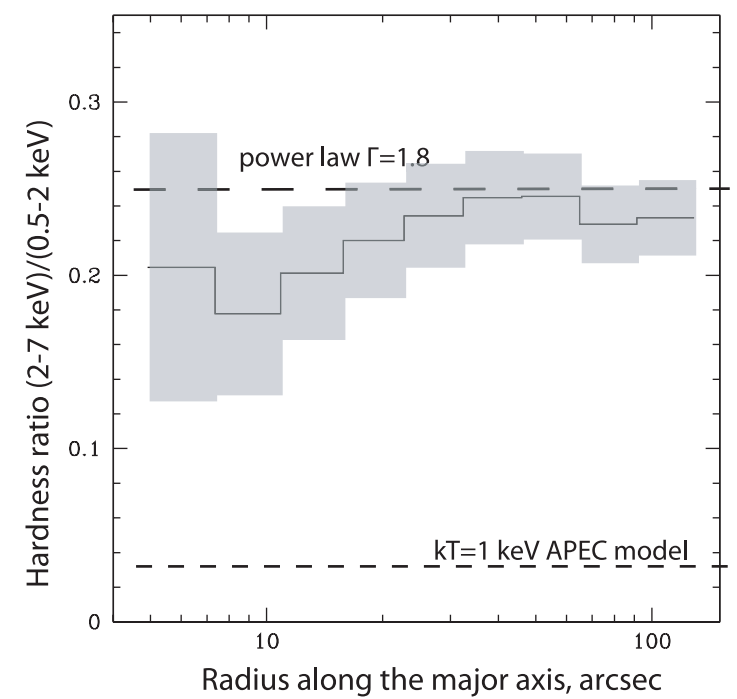

Fig. 3. Ratio of the X-ray fluxes in the energy bands $2-7 \mathrm{keV}$ and $0.5-2 \mathrm{keV}$ within a given radius from the center along the M 32 major axis. The shaded area indicates the $1 \sigma$ uncertainty in this ratio. For comparison shown are the hardness ratios corresponding to two representative spectral models: a power law and an optically thin thermal emission.

consistent (within errors) with being constant over radius from few to $\sim 100^{\prime \prime}$ (Fig. 3). In addition, as Fig. 3 shows, the value of the hardness is charactestic of hard emission (long dashed line) rather than that from soft (thermal) emission (short dashed line).

\subsection{Spectrum}

In Fig. 4 we show the energy spectrum of the unresolved extended X-ray emission. We accumulated this spectrum in the elliptical region with major/minor axes of $62.5^{\prime \prime} / 50^{\prime \prime}$ around the center of M32. This region contains $\sim 70 \%$ of the total optical light (and hence stellar mass) of the galaxy.

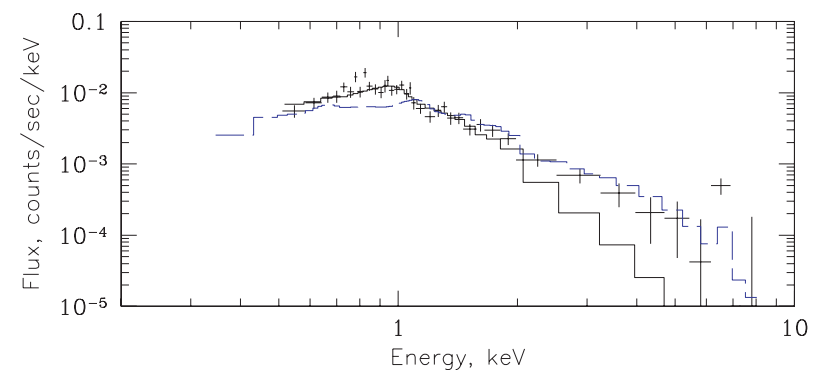

Fig. 4. Spectrum of the unresolved extended emission of M32. The solid and dashed histograms represent optically thin plasma emission models with $k T=1$ and $5 \mathrm{keV}$, respectively. The adopted abundance of elements is 0.03 and 1.0 in the former and latter case, respectively.

A single-temperature fit (APEC model, refs.) to this spectrum in the $0.5-7 \mathrm{keV}$ band is shown in Fig. 4 as the solid histogram. The formal best-fit value of the temperature is $\sim 0.94 \mathrm{keV}$, while the best-fit abundance is extremely low, $\sim 0.04$ of solar. These results are consistent with the values reported by Ho et al. (2003). However, with the increased exposure time available now it is clear that the $k T \sim 1 \mathrm{keV}$ plasma model fails to account for the emission at energies $>2-3 \mathrm{keV}$. Moreover, the hardness of the unresolved X-ray emission of M 32 was already evident from Fig. 3. A much higher temperature $k T \sim 5 \mathrm{keV}$ is needed to describe the data above $>2 \mathrm{keV}$ (blue histogram in Fig. 4), but this model cannot by itself explain the bulk of the soft $\mathrm{X}$-ray emission at energies below $\sim 1 \mathrm{keV}$. Thus, in terms of thermal plasma emission models, a multi-temperature plasma (with the temperature distribution spanning a broad range) is necessary to explain the observed spectrum in the $0.5-7 \mathrm{keV}$ band.

It is interesting to compare the spectrum of the unresolved emission with the cumulative spectrum of the point sources detected by Chandra. The central elliptical region of M 32 introduced above is dominated by the LMXB (probably containing a neutron star) M 32 X-3. The time-averaged X-ray flux of this source is $F_{0.5-7 \mathrm{keV}} \sim 6.8 \times 10^{-13} \mathrm{erg} \mathrm{s}^{-1} \mathrm{~cm}^{-2}$, which translates into a luminosity $L_{0.5-7 \mathrm{keV}} \sim 5 \times 10^{37} \mathrm{erg} \mathrm{s}^{-1}$. In our Galaxy, LMXBs with such luminosities are usually found in the so-called high spectral state, when the optically thick accretion disk extends down to the neutron-star surface. Therefore, most of the X-ray emission in such systems is produced in the optically thick accretion disk and boundary/spreading layer. The spectrum of M 32 X-3 is shown in Fig. 5. Clearly it is very different from the spectrum of unresolved emission.

\subsection{Luminosity}

Assuming a power-law shape of the spectrum with photon in$\operatorname{dex} \Gamma=2.0$, the $2-10 \mathrm{keV}$ diffuse flux from the M32 central elliptical region containing $70 \%$ of the galaxy's optical light (see above) is $F_{2-10 \mathrm{keV}}=(4.4 \pm 0.6) \times 10^{-14} \mathrm{erg} \mathrm{s}^{-1} \mathrm{~cm}^{-2}$ (flux is corrected for the masked out circles around bright point sources). If we fit the spectral data, the best-fit slope parameter is $\Gamma=2.3 \pm 0.4$ and the $2-10 \mathrm{keV}$ flux is $F_{2-10 \mathrm{keV}}=$ $(3.9 \pm 1.2) \times 10^{-14} \mathrm{erg} \mathrm{s}^{-1} \mathrm{~cm}^{-2}$. Assuming a distance of $805 \mathrm{kpc}$ (Mateo 1998), we can then estimate the X-ray luminosity from the whole galaxy as $L_{2-10 \mathrm{keV}}=(4.9 \pm 0.7) \times 10^{36} \mathrm{erg} \mathrm{s}^{-1}$ for $\Gamma=2.0$ and $L_{2-10 \mathrm{keV}}=(4.3 \pm 1.3) \times 10^{36} \mathrm{erg} \mathrm{s}^{-1}$ for $\Gamma=2.3$.

We can estimate the near-infrared luminosity and stellar mass of M 32 from its total $K$-band magnitude of 5.095 (hereafter all optical and near-infrared characteristics of M 32 are adopted from NED unless otherwise noted), applying the 


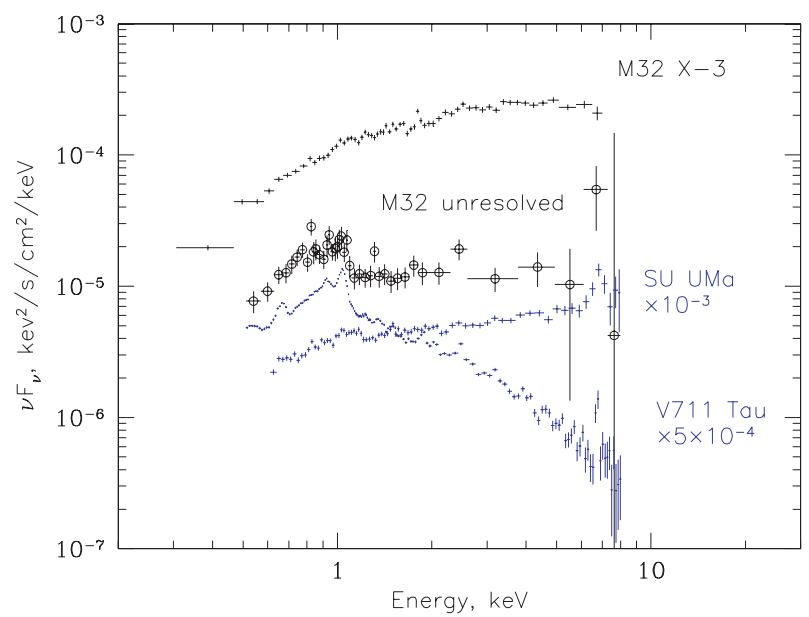

Fig. 5. Spectrum of the bright low-mass X-ray binary M 32 X-3 and the unfolded (through the power-law model with $\Gamma=2.0$ ) spectrum of the unresolved emission of M 32. For illustration we also show the arbitrarily scaled spectra (XMM-Newton/EPIC-MOS data) of a typical coronally active binary star (V711 Tau) and a cataclysmic variable (SU Uma). Sources like these probably make up the bulk of the unresolved emission in M32.

distance modulus of 24.53, correcting for the interstellar extinction of $0.023 \mathrm{mag}$, and using the color-dependent $K$-band massto-light ratio from Bell et al. (2003) for $B-V=0.88$. This results in a total $K$-band luminosity $L_{K}=1.3 \times 10^{9} L_{\odot}$ and a total stellar mass $M_{*}=1.0 \times 10^{9} M_{\odot}$. Using these values, we can determine the emissivity of the diffuse X-ray component per unit stellar luminosity and per unit stellar mass:

$$
\begin{aligned}
& \frac{L_{2-10 \mathrm{keV}}}{L_{K}}=3.3 \pm 1.0 \times 10^{27} \mathrm{erg} \mathrm{s}^{-1} L_{\odot}^{-1}, \\
& \frac{L_{2-10 \mathrm{keV}}}{M_{*}}=4.3 \pm 1.3( \pm 1.3) \times 10^{27} \mathrm{erg} \mathrm{s}^{-1} M_{\odot}^{-1} .
\end{aligned}
$$

The quoted uncertanties derive from the statistical errors on the measured X-ray flux. For the $L_{2-10 \mathrm{keV}} / M_{*}$ ratio we have assumed that an additional uncertainty $\sim 30 \%$ might be associated with the $L_{\mathrm{K}}$ to $M_{*}$ conversion (see e.g. Bell et al. 2003). This systematic uncertainty is given in parentheses.

\section{Discussion}

Apart from the dominant X-ray emission from resolved point sources in M 32, we have clearly detected extended unresolved emission (thus confirming the result of Ho et al. 2003). The data presented above suggest that the cumulative emission of faint, stellar-type X-ray sources in M 32 provides the dominant contribution to this apparently "diffuse" component, similarly to the case of the Milky Way's ridge emission. However, before discussing this scenario we first consider other possibilities to produce diffuse-like X-ray emission as seen by Chandra in M 32.

\subsection{Diffuse emission from hot interstellar gas?}

Let us consider the hypothesis that the detected extended emission originates in a hot diffuse gas pervading M32 and see whether it is consistent with observations.

Assuming a $\beta$-model for the spatial distribution of gas in M32, we can deproject the observed X-ray surface brightness profile and then estimate the total mass of the hot gas within the galaxy.

Approximating the observed surface-brightness profile $S(r)$ at $5^{\prime \prime}<r<120^{\prime \prime}$ by the model (hereafter in this section we assume for simplicity that the gas distribution is spherically symmetric)

$S(r)=S(0)\left[1+\left(\frac{r}{r_{\mathrm{c}}}\right)^{2}\right]^{3 \beta+1 / 2}$,

yields a core radius $r_{\mathrm{c}} \sim 5^{\prime \prime}$ and $\beta \sim 0.34$. Using the emission measure $\left(\sim \int N^{2} \mathrm{~d} V\right)$ of hot (we adopted here $\left.k T=1 \mathrm{keV}\right)$ plasma as seen by Chandra within $50^{\prime \prime}$ radius around the nucleus, we can estimate the mass of the hot gas in the galaxy. The inferred integrated mass of hot gas in the central $50^{\prime \prime}$ is $\sim 0.5 \times 10^{5} M_{\odot}$.

If we now assume that this gas is in hydrostatic equilibrium within the potential well of the galaxy and has a single temperature, we can estimate the total gravitating mass of the galaxy within $\sim 50^{\prime \prime}$ :

$M_{\text {grav }}(<r)=\frac{-k T_{\text {gas }}}{G \mu m_{\mathrm{H}}}\left(\frac{\mathrm{d} \log \rho_{\text {gas }}}{\operatorname{dlog} r}\right)$.

We thus find that the total mass of the galaxy within $50^{\prime \prime}$ is $\sim 10^{10} M_{\odot}$ (assuming a $k T_{\text {gas }}=1 \mathrm{keV}$ ). This value is much higher than the total gravitating mass of M $32\left(7-9.4 \times 10^{8} M_{\odot}\right)$ measured from the velocity dispersion of planetary nebulae (Nolthenius \& Ford 1986) ${ }^{3}$. We have already seen that a single temperature model does not provide a satisfactory description of the X-ray spectrum and a multi-temperature plasma is needed with temperatures ranging from $\sim 1$ up to $\sim 5 \mathrm{keV}$. To make the mass estimate from the hydrostatic equilibrium condition consistent with the one based on planetary nebulae, one would need to assume that the gas has temperature $\sim 0.1 \mathrm{keV}-$ in strong contrast with our spectral measurements. Therefore, we conclude that the hot gas cannot be in hydrostatic equilibrium within the potential well of M 32 .

If we instead assume that there is a constant supply of gas from the stars and this gas is freely escaping the galaxy, we can estimate how many old stars are needed to maintain $\sim 10^{5} \mathrm{M}_{\odot}$ of hot $(\sim 1 \mathrm{keV})$ gas within $\sim 50^{\prime \prime}$. According to different estimates, typical mass ejection rates in elliptical galaxies are $1-3 \times 10^{-11}\left(L_{\mathrm{V}} / L_{\odot, \mathrm{V}}\right) M_{\odot} \mathrm{yr}^{-1}$ (e.g. Faber \& Gallagher 1976; Padovani \& Matteucci 1993; Athey et al. 2002). Therefore, the stars in the central $50^{\prime \prime}$ region of M $32\left(L_{\mathrm{V}} \sim 10^{8} L_{\odot}\right)$ are expected to provide $\dot{M} \sim(1-3) \times 10^{-3} M_{\odot}$ year $^{-1}$ of gas. We should compare this value with the expected mass loss rate of hot gas due to its outflow from the galaxy ( $200 \mathrm{pc}$ in size $)$ at approximately the sound speed $\left(c_{\mathrm{s}} \sim 500 \mathrm{~km} \mathrm{~s}^{-1}\right.$ for a $1 \mathrm{keV}$ gas): $\dot{M} \sim 0.5 \times 10^{5} M_{\odot} /\left(200[\mathrm{pc}] / 400\left[\mathrm{~km} \mathrm{~s}^{-1}\right]\right) \sim 0.1 M_{\odot} \mathrm{year}^{-1}$. We conclude that the scenario where the hot gas is being constantly replenished by the stellar population is also very unlikely.

\footnotetext{
${ }^{3}$ Note that while this value of the total mass of the galaxy is slightly smaller than the stellar mass determined from the $K$-band luminosity of the galaxy $\left(\sim 10^{9} M_{\odot}\right)$, it is consistent with it given the different uncertainties involved in the measurement of both values. On a general note, it was mentioned by Nolthenius \& Ford (1986) that the mass to light ratio of M 32 is consistent with that of globular clusters, therefore it is likely that stars provide the dominant contribution to the total mass of M 32 .
} 
Table 1. X-ray emissivity of the old stellar populations (sources fainter than $\sim 10^{34} \mathrm{erg} \mathrm{s}^{-1}$ in the $2-10 \mathrm{keV}$ band) in M 32 (measured by Chandra) and in the Milky Way.

\begin{tabular}{l|c|c|c}
\hline \hline & $0.5-2 \mathrm{keV}$ & $2-7 \mathrm{keV}$ & $2-10 \mathrm{keV}$ \\
\hline \multicolumn{4}{|c|}{$\mathrm{M} 32^{a}$} \\
$L_{\mathrm{x}} / L_{\mathrm{K}}, 10^{27} \mathrm{erg} \mathrm{s}^{-1} L_{\odot}^{-1}$ & $4.1 \pm 0.6$ & $2.7 \pm 0.8$ & $3.3 \pm 1.0$ \\
$L_{\mathrm{x}} / M_{*}, 10^{27} \mathrm{erg} \mathrm{s}^{-1} M_{\odot}^{-1}$ & $5.4 \pm 0.7( \pm 1.6)$ & $3.5 \pm 1.0( \pm 1.3)$ & $4.3 \pm 1.3( \pm 1.3)$ \\
\hline \multicolumn{4}{c}{$\mathrm{Milky}^{-1}$ Way, GRXE ${ }^{b}($ Revnivtsev et al. 2006) } \\
$L_{\mathrm{x}} / M_{*}, 10^{27} \mathrm{erg} \mathrm{s}^{-1} M_{\odot}^{-1}$ & - & $2.4 \pm 0.4( \pm 1.0)$ & $3.1 \pm 0.5( \pm 1.2)$ \\
\hline \multicolumn{4}{c}{$\mathrm{Milky} \mathrm{Way,} \mathrm{solar} \mathrm{vicinity}^{c}$ (Sazonov et al. 2006) } \\
$L_{\mathrm{x}} / M_{*}, 10^{27} \mathrm{erg} \mathrm{s}^{-1} M_{\odot}^{-1}$ & $9 \pm 3$ & $2.4 \pm 0.6$ & $3.1 \pm 0.8$ \\
\hline
\end{tabular}

\begin{abstract}
${ }^{a}$ The errors quoted in parentheses for the $L_{\mathrm{x}} / M_{*}$ ratio indicate the possible uncertainty in the $L_{\mathrm{K}}$ to $M_{*}$ conversion, which is probably $\sim 30 \%{ }^{b}$ The quoted values were derived assuming that all of the GRXE originates from weak unresolved point sources; these values were recalculated from the 3-20 keV band assuming a power-law spectrum with $\Gamma=2.1$ (Revnivtsev 2003). The errors quoted in parentheses indicate the uncertainty in the assumed mass of the Galactic bulge (see details in Revnivtsev et al. 2006). ${ }^{c}$ The quoted values exclude the contribution of young stars, which is significant in the solar neighborhood (Sazonov et al. 2006). Such sources are expected to be almost completely absent in early-type galaxies like M32, while their overall contribution to the GRXE is unknown. The uncertainty in the fraction of young stars mostly affects the lowest energy bin $0.5-2.0 \mathrm{keV}$; the total cumulative emissivity of all sources (ABs, young stars and CVs) near the Sun in this bin is $\sim 27 \times 10^{27} \mathrm{erg} \mathrm{s}^{-1} M_{\odot}^{-1}$. The $0.5-2 \mathrm{keV}$ emissivity was estimated by multiplying the original $0.1-2.4 \mathrm{keV}$ value by 0.7 (see Sazonov et al. 2006, for details). The $2-7 \mathrm{keV}$ emissivity was converted from the $2-10 \mathrm{keV}$ band assuming a power-law spectrum with $\Gamma=2.1$, as for the GRXE.
\end{abstract}

\subsection{Emission from unresolved sources - the old stellar population}

We have demonstrated that the hot insterstellar gas model clearly fails to account for the observed diffuse emission of M 32. We are now turning to the hypothesis that the bulk of the diffuse emission is due to weak sources. Given the excellent sensitivity of the Chandra dataset for M32, we were able to resolve all X-ray sources with luminosities higher than $L_{\mathrm{x}} \sim$ $8 \times 10^{33} \mathrm{erg} \mathrm{s}^{-1}$. However, still lower luminosity sources remain undetected and could provide the unresolved X-ray flux should their space density be sufficiently high.

In this regard we note that stellar-type X-ray sources with luminosities below $10^{34} \mathrm{erg} \mathrm{s}^{-1}$ are abundant in our Galaxy, in particular in its old stellar population, the majority of these sources being cataclysmic variables (CVs) and active binaries (ABs) (see e.g. Vaiana 1981; Sazonov et al. 2006). Therefore, for a relatively old galaxy with very low star formation rate, like M32, one should also expect a considerable X-ray luminosity from the numerous CVs and ABs. From the luminosity and other properties of the GRXE (e.g. Revnivtsev et al. 2006) and from direct measurements of the luminosity function of sources in the solar neighborhood (Sazonov et al. 2006), the combined 2-10 keV emissivity of CVs and ABs has been estimated as $L_{\mathrm{x}} / M_{*} \sim 2-4.5 \times 10^{27} \mathrm{erg} \mathrm{s}^{-1} M_{\odot}$. The value $(4.3 \pm 1.3 \pm 1.3) \times 10^{27} \mathrm{erg} \mathrm{s}^{-1} M_{\odot}$ obtained from observations of M 32 falls exactly in this range. This, together with the similarity of the X-ray and near-infrared surface brightness maps, strongly suggests that in M32 we are dealing with essentially the same phenomenon as the GRXE in our Galaxy, namely the collective $\mathrm{X}$-ray emission from millions of faint, stellar-type sources.

Within this scenario, one should expect the spectrum of the M 32 diffuse X-ray emission to be complex. Indeed, although we expect all of the unresolved emission to originate in opticallythin thermal plasmas, these, in contrast to e.g. the case of intracluster gas in galaxy clusters, should be characterized by a broad range of temperatures. Indeed, active stellar coronae typically have temperatures in the range $0.1-3 \mathrm{keV}$, while the $\mathrm{X}$-ray emitting regions in $\mathrm{CV}$ s have much higher temperatures up to $20-30 \mathrm{keV}$. To illustrate this point we show in Fig. 5 typical spectra of an AB (V711 Tau) and a CV (SU UMa). It is evident that a combination of such spectra could well resemble the observed spectrum of the M 32 X-ray halo. Specifically, the $0.8-1 \mathrm{keV}$ peak would be mostly due to $\mathrm{ABs}$, while the harder X-ray tail due to both ABs and CVs. We do not attempt to carry out a more quantitative modelling of the observed spectrum along these lines, since there are significant uncertainties in our understanding of the dependence of stellar coronal temperatures on luminosity, stellar type and age, as well as of the soft $\mathrm{X}$-ray spectra of different subclasses of CVs.

Table 1 summarizes several independent estimates of the X-ray emissivity of the old stellar population per unit stellar mass in different energy bands: $0.5-2 \mathrm{keV}, 2-7 \mathrm{keV}$, and $2-10 \mathrm{keV}$. These estimates include the current one based on M32, for GRXE and for the solar neighborhood.

We note that despite the excellent agreement at energies above $2 \mathrm{keV}$, there is a hint that the local (near the Sun) soft X-ray $(0.5-2 \mathrm{keV})$ emissivity, even excluding young (age $\ll$ $1 \mathrm{Gyr}) \mathrm{stars}^{4}$, is somewhat higher than the value obtained for M32. Since the soft X-ray emissivity is dominated by ABs, this difference may indicate an intrinsic difference in the old stellar populations of M 32 and our local environment. We might also anticipate that galaxies with a star formation history similar to that of our Galaxy should be charactered by a cumulative emissivity closer to that obtained near the Sun than to that obtained for M 32 .

It is well-known that LMXBs can make an important contribution to the X-ray flux of an elliptical galaxy, especially for a gas poor one. If the LMXBs are bright enough to be directly resolved, then their contribution can be accounted for and in the remaining unresolved flux the role of weaker objects will be higher. The dependence of the cumulative X-ray emissivity of the unresolved stellar population as a function of sensitivity (minimum luminosity of resolved sources) is shown in Fig. 6. The shaded areas are the results of integration of the luminosity function of LMXBs determined by Gilfanov (2004) with the added cumulative emissivity of $\mathrm{ABs}$ and $\mathrm{CV}$ s adopted from Sazonov et al. (2006, see also Table 1). From this figure it is clear that if sources with luminosities $L_{0.5-7} \mathrm{keV} \geq 10^{35-36} \mathrm{erg} \mathrm{s}^{-1}$

\footnotetext{
4 Inclusion of young stars would increase the local emissivity even further, up to $L_{0.5-2 \mathrm{keV}} / M \sim 27 \times 10^{27} \mathrm{erg} \mathrm{s}^{-1} M_{\odot}^{-1}$.
} 


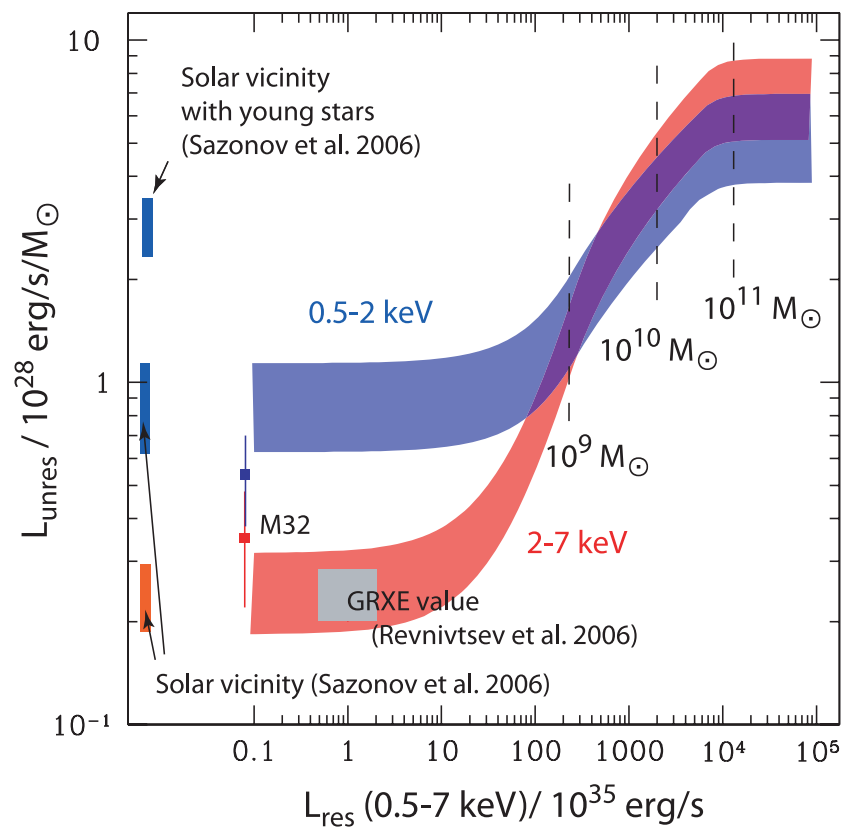

Fig. 6. Cumulative emissivity of unresolved discrete sources as a function of the source detection threshold in a given observation. The shaded areas are derived for two energy bands by integrating the luminosity function of LMXBs from Gilfanov (2004) and adding the cumulative emissivity of fainter X-ray emitting systems from Sazonov et al. (2006, see also Table 1). The widths of these regions were fixed at $30 \%$ to emphasize the possible uncertainties in the stellar mass estimates involved in the measurements. Also shown are values obtained from studies of the Galactic ridge X-ray emission (Revnivstev et al. 2006), Solar vicinity (Sazonov et al. 2006) and M 32 galaxy (this work). The two lower emissivity estimates from Sazonov et al. (2006) exclude the contribution of young stars. Dashed lines mark several masses of galaxies for which on average one LMXB with a $0.5-7 \mathrm{keV}$ luminosity higher than a given value is expected to be found.

can be resolved, the unresolved emission will be dominated by CVs and ABs. It is worth emphasizing that in the $0.5-2 \mathrm{keV}$ energy band the contrast between the cumulative contributions of LMXBs and CVs/ABs is much smaller than in the harder $2-10 \mathrm{keV}$ band. Moreover, bright LMXBs are rare objects and the probability of finding them in a small galaxy is low. In Fig. 6 the dashed lines mark the masses of galaxies in which on average one LMXB with a $0.5-7 \mathrm{keV}$ luminosity higher than a given value is expected to be found. Thus for a typical gas poor galaxy with mass less then $10^{10} M_{\odot}$, which will typically miss very bright sources with luminosities $>10^{38} \mathrm{erg} \mathrm{s}^{-1}$, the contribution of $\mathrm{CVs} / \mathrm{ABs}$ to the total X-ray emissivity of the galaxy can be $\sim 30 \%$ or higher.

While the 3-20 keV cumulative spectrum of the old stellar population in our Galaxy has been reliably measured (Revnivtsev et al. 2006), it is very difficult to extend this analysis to energies below $\sim 3 \mathrm{keV}$ because of the strong interstellar photoabsorption in the Galaxy. M 32 data do not suffer from such strong absorption. Therefore, assuming that the X-ray emissivities of the old stellar populations in our Galaxy and in M 32 are similar, we can combine all of available data to build the cumulative spectrum of the stellar populations in a broad energy range.

For this purpose we use our Chandra results on M32 and RXTE/PCA (Revnivtsev et al. 2006) and INTEGRAL/IBIS (Krivonos et al. 2006) on the Milky Way. The resulting broad band spectrum of the cumulative emission of weak X-ray
Spectrum of stellar population per unit stellar mass

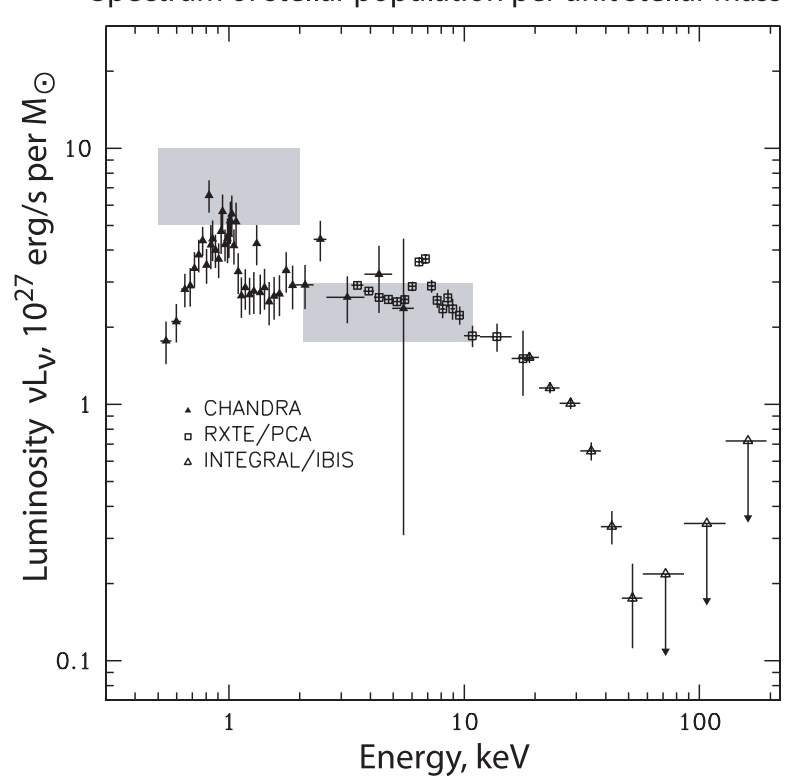

Fig. 7. Broad-band spectrum of the weak X-ray source population of a typical old galaxy, compiled from data for the GRXE (3-20 keV RXTE/PCA, Revnivtsev et al. 2006, 20-200 keV - INTEGRAL/IBIS, Krivonos et al. 2006) and for M 32 (0.5-7 keV - Chandra, this work). For M 32 a mass of $M=10^{9} M_{\odot}$ is assumed. No fiducial factors were applied to build this spectrum over almost three orders of magnitude in energy. Gray rectangles represent the cumulative emissivities of X-ray sources, excluding young stars, in the Solar neighborhood (Sazonov et al. 2006, see Table 1).

sources is shown in Fig. 7. In the high-energy end of this spectrum (>10-20 keV), intermediate polars dominate (Revnivtsev et al. 2006; Krivonos et al. 2006), while at low energies coronally active stars contribute most significantly. The gray rectangles show data obtained for the Solar vicinity (Sazonov et al. 2006). Note that the $0.5-2 \mathrm{keV}$ emissivity calculated for the Solar vicinity is higher than the corresponding value for M32, even though the former already excludes the contribution of young stars. As discussed above, this tentative difference in the emissivities in the $0.5-2 \mathrm{keV}$ band may reflect the younger age of stars near the Sun compared to M 32.

\subsection{Predictions and future work}

An obvious prediction from our study is that any elliptical galaxy with an optical luminosity $L_{\mathrm{opt}}$ should have a minumum level of X-ray luminosity $L_{\mathrm{x}} \sim 3-5 \times 10^{39}\left(M_{*} / 10^{12} M_{\odot}\right) \mathrm{erg} \mathrm{s}^{-1}$ even if it is very gas poor and all bright LMXBs have been resolved.

6.7 keV emission line. We have demonstrated that the unresolved X-ray halo in M32 can be satisfactorily explained as the cumulative emission from $\mathrm{CVs}$ and $\mathrm{ABs}$, similar to the GRXE. In this regard we note that a salient characteristic feature of the GRXE is the strong complex of emission lines at $6.4 \mathrm{keV}, 6.7 \mathrm{keV}$, and $6.9 \mathrm{keV}$, due to highly ionized iron (6.7 and $6.9 \mathrm{keV}$ lines) and fluorescenece of iron in a relatively cold medium (6.4 keV). One could then expect M 32 to exhibit similar strong emission lines (with a combined equivalent width of up to $\sim 600 \mathrm{eV}$, see e.g. Koyama et al. 1986). The current statistics from Chandra do not allow us to claim the presence of these lines, although we do see an indication of iron emission. In 
order to securely detect the $6.7 \mathrm{keV}$ line with Chandra, one would need $\sim 1$ Ms worth of observations.

Absence of "X-ray cavities". If our hypothesis for the compactsource nature of the unresolved emission in M 32 is correct, one could anticipate there should be no cavities produced in this $\mathrm{X}$-ray halo by the plasma pressure of radio-bubbles, as is often observed in elliptical galaxies or clusters (e.g. Bohringer et al. 1995; Churazov et al. 2001; Bîrzan et al. 2004). Unlike trully diffuse gas, relativistic plasma should have no impact on the X-ray emission associated with the stars (the same is true for LMXBs). While in M 32 there is no evidence for any powerful relativistic plasma, other galaxies could provide an opportunity to test this prediction. We note however that the surface brightness of the $\mathrm{X}$-ray emission due to old stars is rather low and it may be problematic to detect the old stellar component unambiguosly in gas rich and/or more distant galaxies.

\section{Conclusions}

We have shown that the unresolved X-ray halo in the M 32 dwarf elliptical galaxy can be best explained by GRXE-like emission, i.e. cumulative emission from cataclysmic variables and coronally active stars. We have combined the spectra of the M 32 diffuse emission and of the GRXE to obtain a broad-band X-ray spectrum of the old stellar population in our and other galaxies. We also derived predictions of the unresolved X-ray emissivity (X-ray luminosity per solar mass) of faint, stellar X-ray sources, in both soft $(0.5-2 \mathrm{keV})$ and hard (2-7 keV) bands, as a function of observational sensitivity (minimum luminosity of resolved sources) (Fig. 6) which is critical for understanding the unresolved emission from galaxies.

Acknowledgements. This research made use of data obtained from the High Energy Astrophysics Science Archive Research Center Online Service, provided by the NASA/Goddard Space Flight Center. This publication makes use of data products from the Two Micron All Sky Survey, which is a joint project of the University of Massachusetts and the Infrared Processing and Analysis Center/California Institute of Technology, funded by the National Aeronautics and Space Administration and the National Science Foundation. This work was supported by DFG-Schwerpunktprogramme (SPP 1177).

\section{References}

Athey, A., Bregman, J., Bregman, J., Temi, P., \& Sauvage, M. 2002, ApJ, 571, 272

Bell, E. F., McIntosh, D. H., Katz, N., \& Weinberg, M. D. 2003, ApJS, 149, 289 Bîrzan, L., Rafferty, D. A., McNamara, B. R., Wise, M. W., \& Nulsen, P. E. J. 2004, ApJ, 607, 800

Bohringer, H., Nulsen, P. E. J., Braun, R., \& Fabian, A. C. 1995, MNRAS, 274, L67

Canizares, C. R., Fabbiano, G., \& Trinchieri, G. 1987, ApJ, 312, 503

Churazov, E., Brüggen, M., Kaiser, C. R., Böhringe, H., \& Forman, W. 2001, ApJ, 554, 261

Cohen, M., Wheaton, W. A., \& Megeath, S. T. 2003, AJ, 126, 1090

Dwek, E., Arendt, R. G., Hauser, M. G., et al. 1995, ApJ, 445, 716

Fabbiano, G., \& White, N. 2003, in Compact Stellar X-ray Sources, ed. W. Lewin, \& M. van der Klis (Cambridge University Press)

[arXiv: astro-ph/0307077]

Faber, S. M., \& Gallagher, J. S. 1976, ApJ, 204, 365

Forman, W., Jones, C., \& Tucker, W. 1985, ApJ, 293, 102

Fukazawa, Y., Botoya-Nonesa, J. G., Pu, J., Ohto, A., \& Kawano, N. 2006, ApJ, 636, 698

Gilfanov, M. 2004, MNRAS, 349, 146

Ho, L. C., Terashima, Y., \& Ulvestad, J. S. 2003, ApJ, 589, 783

Kent, S. M. 1987, AJ, 94, 306

Koyama, K., Makishima, K., Tanaka, Y., \& Tsunemi, H. 1986, PASJ, 38, 121

Krivonos, R., Revnivtsev, M., Churazov, E., et al. 2006, A\&A, 463, 957

Loewenstein, M., Hayashida, K., Toneri, T., \& Davis, D. S. 1998, ApJ, 497, 681

Mateo, M. L. 1998, ARA\&A, 36, 435

McCammon, D., \& Sanders, W. T. 1990, ARA\&A, 28, 657

Nolthenius, R., \& Ford, H. 1986, ApJ, 305, 600

Ottmann, R., \& Schmitt, J. H. M. M. 1992, A\&A, 256, 421

Padovani, P., \& Matteucci, F. 1993, ApJ, 416, 26

Revnivtsev, M. 2003, A\&A, 410, 865

Revnivtsev, M., Sazonov, S., Gilfanov, M., Churazov, E., \& Sunyaev, R. 2006, A\&A, 452, 169

Revnivtsev, M., Vikhlinin, A., \& Sazonov, S. 2007, A\&A, 473, 857

Sazonov, S., Revnivtsev, M., Gilfanov, M., Churazov, E., \& Sunyaev, R. 2006, A\&A, 450, 117

Schmitt, J. H. M. M., Collura, A., Sciortino, S., et al. 1990, ApJ, 365, 704

Vaiana, G. S. 1981, SSRv, 30, 151

Vikhlinin, A., McNamara, B. R., Forman, W., et al. 1998, ApJ, 502, 558

Vikhlinin, A., Markevitch, M., Murray, S. S., et al. 2005, ApJ, 628, 655

Welch, G. A., \& Sage, L. J. 2001, ApJ, 557, 671

Worrall, D. M., \& Marshall, F. E. 1983, ApJ, 267, 691

Worrall, D. M., Marshall, F. E., Boldt, E. A., \& Swank, J. H. 1982, ApJ, 255, 111 